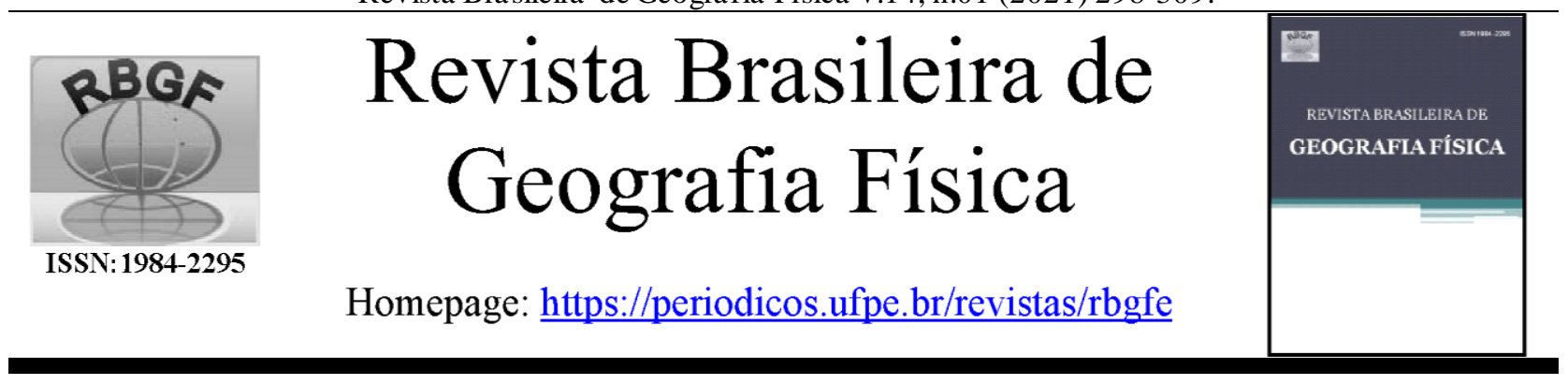

\title{
Ciclone Michael: gênese e transição extratropical
}

\author{
RaquelGonçalves Pereira ${ }^{1}$, Aline Aquino de Araujo ${ }^{2}$, Michelle Simões Reboita ${ }^{3}$
}

${ }^{1}$ Graduanda deCiências Atmosféricas, Instituto de Recursos Naturais, Universidade Federal de Itajubá, Av. BPS, 1303, Pinheirinho - Caixa Postal 50, CEP 37500 903, Itajubá, Minas Gerais. (35) 98899-9087.raquelgpereira10@ gmail.com. ${ }^{2}$ Graduanda de Ciências Atmosféricas, Instituto de Recursos Naturais, Universidade Federal de Itajubá, Av. BPS, 1303, Pinheirinho - Caixa Postal 50, CEP 37500 903, Itajubá, Minas Gerais. (12) 99653-1412. alineaaraujo@outlook.com. ${ }^{3}$ Profa. Dra. do Instituto de Recursos Naturais, Universidade Federal de Itajubá, Av. BPS, 1303, Pinheirinho - Caixa Postal 50, CEP 37500903, Itajubá, Minas Gerais. (35)9102-5193. reboita@gmail.com.

Artigo submetido em 29/04/2020 e aceite 04/02/2021

\section{R E S U M O}

Os ciclones de escala sinótica são responsáveis por grandes mudanças no tempo das regiões onde atuam. Nos últimos a nos tem aumentado o número de sistemas tropicais severos. Por exemplo, o ciclone Michael, ocorrido em outubro de 2018, causou muitos danos nos Estados Unidos (U\$25 bilhões em prejuízo), inclusive 16 óbitos. Diante desse contexto, o objetivo do presente estudo é a análise sinótica da gênese e transição extratropical do ciclone Michael. Michael teve gênese no mar do Caribe, no dia 6 de outubro de 2018, associada a uma perturbação ciclônica em baixos níveis da atmosfera. O sistema chegou a categoria 5 na escala de Saffir-Simpson no dia 10 de outubro; já no dia 11 de outubro transicionou para ciclone extratropicale decaiu no dia 18 de outubro. A análise sinótica mostra que transição extratropical ocorre à medida que o sistema intera ge com uma região de intenso gradiente horizontalde temperatura do ar.

Palavras-chave: ciclone, análise sinótica, transição extra tropical.

\section{Cyclone Michael: genesis and extratropical transition}

\section{A B S T R A C T}

Synoptic-scale cyclones are responsible for major changes in the weather in the regions where they act. In recent years the number of severe tropical systems has increased. For example, cyclone Michael, which occurred in October 2018, caused a lot of damage in the United States (\$25 billion in damage) including 16 deaths. Given this context, the objective of the present study is the synoptic analysis of the genesis and extratropicaltran sition of cyclone Michael. Michael had genesis in the Caribbean Sea on October 6, 2018, a ssociated with a cyclonic disturbance in low levels of the atmosphere. The system reached category 5 on the Saffir-Simpson scale on 10 October; on the 11 October it transitioned to an extratropicalcyclone and decayed on the 18 October. The synoptic analysis shows that extratropicaltransition occurs as the system interacts with a region of intense horizontalair temperature gradient.

Keywords: cyclone, synoptic analysis, extratropicaltransition.

\section{Introdução}

Os ciclones tropicais são sistemas de baixa pressão, formados sobre os oceanos, e que apresentam convecção persistente e organizada. A fonte de energia dos ciclones tropicais é basicamente a evaporação dos oceanos. Por esse motivo, quando chegam ao continente, há supressão de energia e, em geral, os sistemas se dissipam (Charney e Ellassen, 1964; Reboita et al., 2017a).

Os ciclones tropicais se deslocam para oeste ou noroeste, seguindo o padrão da circulação geral da atmosfera nos trópicos. Quanto à estrutura vertical desses sistemas, eles possuem núcleo mais quente do que suas cercanias que é mais intenso em médios e altos níveis da atmosfera (Reboita et al., 2017a,b) e são sistemas com aparência simétrica tanto na nebulosidade quanto nas demais variáveis atmosféricas (Gray e Brody, 1967; Frank, 1977; Lim et al., 2015; Reboita et al., 2017a). Possui um tubo de vorticidade relativa ciclônica que é alinhada na vertical, o que confere a característica de sistema barotrópico aos ciclones tropicais. 
Segundo o National Hurricane Center (NHC, 2020), os ciclones tropicais podem ser classificados de acordo com a intensidade dos ventos em depressão tropical quando os ventos atingem até $61 \mathrm{~km} / \mathrm{h}$, tempestade tropical quando a intensidade dos ventos está entre 62 e $118 \mathrm{~km} / \mathrm{h}$ e quandoa intensidade dos ventos é superior ou igual a $119 \mathrm{~km} / \mathrm{h}$ a classificação é dada em função da localização do ciclone. Dessa forma, são chamados de furacão se a gênese ocorrer na bacia do oceano Atlântico e Pacífico Sul, tufão quando se originam no Pacifico Oeste e de ciclones quando se formam no oceano Índico.

De acordo com a magnitude dos ventos sustentados (intensidade do vento medida num dado intervalo), os ciclones que atingem a categoria de furacão podem ainda ser classificados numa escala que varia entre 1 e 5, chamada de Escala Saffir-Simpson (NHC, 2020). A categoria 1 da escala refere-se a ventos entre 119 e $153 \mathrm{~km} / \mathrm{h}$, a categoria 2 são ventos de 154 a $177 \mathrm{~km} / \mathrm{h}$, categoria 3 corresponde a 178 e $209 \mathrm{~km} / \mathrm{h}$, a categoria 4 compreende ventos de 210 a $249 \mathrm{~km} / \mathrm{h}$ e a categoria 5 é a mais severa, com ventos mais intensos que $250 \mathrm{~km} / \mathrm{h}$.

Os ciclones tropicais podem se dissipar ou evoluírem (transicionar) para outro tipo de ciclone (Hart et al., 2006; Ritchiee Esberry, 2006). Quando atingem as latitudes médias, podem adquirir características de sistemas extratropicais e o processo recebe o nome de transição extratropical. A transição ex tratropical ocorre devido à mudança na baroclinia (aumento) (Takamurae Wada, 2020). Uma vez que o sistema se move em direção ao polo, encontra um ambiente com gradientes horizontais de temperatura do ar mais intensos que modulam o ciclone tropical em extratropical (Reboita et al., 2017a).

Em outubro de 2018, uma perturbação ciclônica se formou sobre o setor centro-oeste do mar do Caribe, onde se acoplou aos remanescentes da tempestade tropical Kirk entre os dias 1 à 2 de outubro (Beven II et al., 2019). Segundo Beven II et al. (2019), uma onda tropical, que se deslocava de leste para oeste, interagiu com a perturbação ciclônica favorecendo a convergência em superfície e, consequentemente, a intensificação da perturbação ciclônica no sudoeste da Jamaica em 3 de outubro. No dia seguinte, a perturbação ciclônica migrou para oeste-sudoeste, atingindo o nordeste de Honduras. Em 6 de outubro, o ciclone se configura sobre o interior da América Central (Beven II et al., 2019).

No dia 7 de outubroo sistemase intensifica sendo classificado como tempestade tropical (ventos com intensidade sustentada de $61 \mathrm{~km} / \mathrm{h}$ ), recebendo o nome Michael; em 8 de outubro, Michael já era considerado um furacão. Sua intensidade continuou aumentando, atingindo a categoria 3 da escala Saffir-Simpson nas proximidades da costa do Golfo da Flórida - EUA (Beven II et al., 2019).

Segundo Beven II et al. (2019), antes de chegar ao Golfo da Flórida, Michael deslocou-se até passar a Base da Força Aérea de Tyndall (AFB), na Flórida Panhandle no dia 10 de outubro, onde os ventos máximos sustentados chegavam à $250 \mathrm{~km} / \mathrm{h}$ - categoria 5 da escala Saffir-Simpson, o que causou muitos problemas na região costeira.

De acordo com Beven II et al. (2019), Michel não se dissipou ao adentrar o continente, seguiu sua trajetória, passando pela Geórgia no dia 10 de outubro, onde perdeu intensidade e foi rebaixado à categoria de tempestade tropical com ventos com intensidade máxima de $185 \mathrm{~km} / \mathrm{h}$. No 11 de outubro migrou para as Carolinas, deixando um rastro de destruição. Ao passar pela Carolina do Norte, iniciou a transição extratropical, onde a pressão central diminuiu novamente e os ventos se intensificaram; já no dia 12 de outubro a transição foi concluída. Nesse mesmo período, Michael adquiriu trajetória para leste-nordeste, passando pelo norte de Raleigh, Carolina do Norte e depois atravessou a área de Norfolk, Virgínia, chegando ao Atlântico ocidental ainda no dia 12 de outubro. No dia 13 de outubro o ciclone localizava-se sobre o oceano ao sul da Nova Escócia e Newfoundland. Após esse período, a baixa enfraqueceu (Beven II et al., 2019).

Os ciclones tropicais são responsáveis por danos materiais e perda de vidas por causarem chuvas torrenciais, inundações, ventos fortes, e aumento das ondas e nível do mar (Anthes, 1982; Wang e Wu, 2004). Michael foi responsável por 16 mortes e cerca de US \$ 25 bilhões em danos nos Estados Unidos. Antes de atingir os Estados Unidos, o ciclone também causou prejuízos no oeste de Cuba quando era um furacão de categoria 2 (Beven II et al., 2019).

Diante do exposto, o objetivo deste estudo é descrever as características sinóticas da gênese e transição extratropical do ciclone Michael. A relevância desse estudo está no fato de contribuir para o entendimento dos processos físicos associados aos ciclones tropicais, principalmente durante a transição.

\section{Material e métodos}

Dados da reanálise ERA5

Para realizar o estudo sinótico do ciclone Michael, as variáveis consideradas foram as componentes zonal (u) e meridional (v) do vento,

Pereira, R. G., Araujo, A. A., Reboita, M. S. 
altura geopotencial, temperatura do ar e vorticidade relativa em 17 níveis verticais; pressão ao nível médio do mar, fluxo de calor sensível e latente, temperatura da superfície do mar e vento a $10 \mathrm{~m}$ nos horários das 0000, 0600, 1200 e 1800 UTC entre os dias 05 a 12 de outubro de 2018 , na área entre $0^{\circ} \mathrm{e} 70^{\circ} \mathrm{N}$ e $100^{\circ} \mathrm{e} 10^{\circ} \mathrm{W}$. Os dados foram obtidos da reanálise ERA5 (Copernicus Climate Change Service-C3S, 2020), que possui resolução horizontal de $0.25^{\circ} \times 0.25^{\circ}$.

\section{Dados de satélite}

Foram utilizados dados do GridSat (Gridded Satellite - National Climatic Data Center- NCDC, 2020) da NOAA (National Oceanic and Atmospheric Administration). Para este estudo foram utilizadas informações do canal infravermelho (IR - aproximadamente $11 \mu \mathrm{m}$ ). Os dados são disponibilizados em intervalo de 3 horas e possuem resolução horizontal de 0,07 graus. Mais informações sobre esse conjunto de dados podem ser encontradas em Knapp et al. (2011).

\section{Análise Sinótica}

A primeira etapa do estudo consiste em definir a trajetória do ciclone. Essa foi baseada em dados de pressão ao nível médio do mar. Para cada horário sinótico foram plotadas as isóbaras com intervalos de $3 \mathrm{hPa}$. O horário em que apareceu a primeira isóbara fechada foi considerado como ciclogênese. Então a posição (latitude e longitude) e o valor da pressão central do sistema são registradas destetempo ao decaimento (quando não há mais isóbaras fechadas). A trajetória do ciclone é mostrada na primeira seção dos resultados.

Após foram construídos os campos das variáveis atmosféricas listadas abaixo para cada horário sinótico ao longo do ciclo de vida do sistema:

- Pressão ao nível médio do mar, intensidade do vento em $250 \mathrm{hPa}$, e espessura da camada 500/1000 $\mathrm{hPa}$, que é a diferença da altura geopotencial entre duas superfícies de pressão constante e que é proporcional à média da temperatura do ar na camada;

- Temperatura do ar em $850 \mathrm{hPa}$ e vetores do vento em $850 \mathrm{hPa}$;

- Perfil vertical da vorticidade relativa, variável que indica a rotação dos sistemas atmosféricos;

- Perfil vertical do desvio zonal da temperatura do ar e da altura geopotencial, fixada a latitude do centro do ciclone, o desvio zonal é definido como a diferença entre a temperatura do ar (ou altura geopotencial) de uma dada camada (altura) e a média da camada;
- Isotermas em 850 hPa e pressão ao nível médio do mar;

- Fluxos de calor latente e sensível e pressão ao nível médio do mar;

- Cisalhamento vertical do vento entre 200 e 850 $\mathrm{hPa}$, que é a diferença entre a intensidade do vento entre esses dois níveis, e pressão ao nível médio do mar;

- Temperatura de brilho e vento a 10 metros.

Com base nesses campos foram selecionados alguns horários que representam as principais fases do ciclo de vida do Michel:

- Pré-ciclogênese: período que antecede a formação do ciclone;

- Ciclogênese: quando as isóbaras aparecem fechadas pela primeira vez;

- Maturidade da fase tropical: quando o sistema é bem intenso no campo da vorticidade relativa;

- Transição extratropical: quando a fase tropical se desconfigura e o ciclone passa a mostrar forma similar a do modelo conceitual de ciclone extratropical de Bjerknes e Solberg (1922).

A Tabela 1 mostra a data e hora sinótica de cada fase do ciclo de vida do ciclone, enquanto a descrição sinótica é apresentada na seção resultados.

Tabela 1. Data e horário sinótico das fases Ciclone Michael.

\begin{tabular}{c|c}
\hline Fase & Data \\
\hline Pré- Ciclogênese & $12 Z 050 C T 2018$ \\
\hline Ciclogênese & 00Z06OCT2018 \\
\hline Desenvolvimento & $18 Z 080 C T 2018$ \\
\hline Maturidade da Fase Tropical & $12 Z 100 C T 2018$ \\
\hline \multirow{2}{*}{ Transição Extra tropical } & $18 Z 110 C T 2018$ \\
\cline { 2 - 2 } & 00Z12OCT2018 \\
\hline \multirow{2}{*}{ Fase Extratropical } & $12 Z 120 C T 2018$ \\
\cline { 2 - 2 } & $18 Z 120 C T 2018$ \\
\hline
\end{tabular}

\section{Resultados e discussão}

\section{Trajetória Ciclone Michael}

A trajetória mostradana Figura 1 foi obtida registrando-se a latitude e longitude central do ciclone Michael, desde sua ciclogênese até o decaimento já na fase extratropical. A gênese do Michael ocorreu no dia 06 de outubro de 2018 às $0000 \mathrm{UTC}$, em aproximadamente $17^{\circ} \mathrm{Ne} 85^{\circ} \mathrm{W}$ no mar do Caribe e a transição extratropical foi concluída às 0000 UTC do dia 12 de outubro. A transição extratropical começou quando Michael se deslocou para a Carolina do Norte, apresentando queda na pressão e aumento da intensidade dos 
ventos, como será detalhado nas próximas seções. Às 1200 UTC do dia 12, Michael inicia sua fase extratropical.

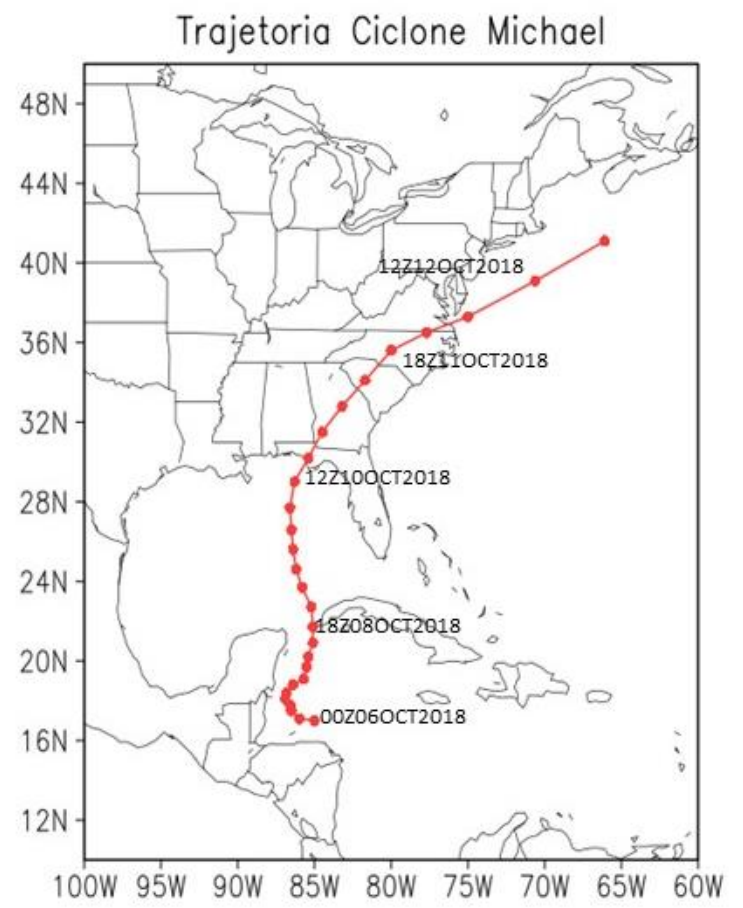

Figura 1. Trajetória do ciclone Michael desde a ciclogênese até o decaimento, que ocorre já na fase extratropical.

\section{Ciclo de Vida do ciclone Michael}

Pré-Ciclogênese

Nessa parte do estudo é realizada uma análise sinótica do ciclone com base nas datas definidas na Tabela 1. A gênese dos ciclones tropicais está associada com a energia que vem do processo de evaporação dos oceanos (Anthes, 1982; Bister e Emanuel 1998), relacionado com a TSM e com os fluxos de calor latente e sensível. O ambiente em que ocorrerá a gêneseé caracterizado, no dia 05 , por temperatura da superfície do mar (TSM) de $29^{\circ} \mathrm{C}$ (Figura não mostrada), por fluxos de calor total (sensível e latente) direcionados da superfície oceânica para a atmosfera de $\sim 50 \mathrm{~W} \mathrm{~m}^{-2}$ (Figura 2a), cisalhamento vertical do vento de $\sim 0$ $\mathrm{m} / \mathrm{s}$ (Figura 3a), local da gênese distante do equador em cerca de $5^{\circ}$ a $6^{\circ} \mathrm{e}$ fraca vorticidade relativa ciclônica $\left(10 \mathrm{~s}^{-1}\right)$ em baixos níveis da atmosfera (nível de $980 \mathrm{hPa}$ ) na região em que o ciclone se forma (Figura 4a). De acordo com Gray (1968), as características apresentadas são condições necessárias, mas não suficientes para a ciclogênese tropical. Isso devido ao fato de que todas as características apresentadas podem aparecer e, mesmo assim, um sistema tropical pode não ocorrer. Para existir a ciclogênese é necessário que a convecção seja mantida e isso ocorre quando a anomalia de vorticidade ciclônica é sustentada e intensificada em superfície e, para isso, em geral, há o suporte de algum outro distúrbio atmosférico. No caso do Michael havia uma onda em $850 \mathrm{hPa}$ de leste para oeste que ajudou a manter a convergência e anomalia de vorticidade ciclônica em superfície (Figura 5a). Diferentemente do ambiente pré-ciclone extratropical, no tropical não há gradientes horizontais de temperatura do ar (Figura 6a) e nem advecção horizontal de temperatura em superfície (Figura 7a). Nas imagens de satélite do canal infravermelho (temperatura de brilho), na fase pré-ciclogenética, já é possível ver nebulosidade nas regiões onde o ciclone formará (Figura 8a), porém com forma assimétrica. Cores mais brancas indicam nuvens mais espessas e com topos frios.

\section{Ciclogênese}

Como as condições ambientais necessárias para a ciclogênese permaneceram do estágio préciclogênese até o atual, o distúrbio pré-existente se organizou num ciclone tropical. A pressão central decresceu e os ventos em superfície intensificaram (Figura 9b). A queda na pressão é evidente através do fechamento das isóbaras. Assim, o dia 6 de outubro às 0000 UTC foi definido como ciclogênese, pois foi quando as isóbaras apareceram fechadas pela primeira vez, com núcleo de 1008 hPa (Figura 9b).Além disso, a vorticidade relativa ciclônica mostra assinatura no perfil vertical (Figura 4b). Nota-se fraco cisalhamento vertical do vento na região ciclônica $(5 \mathrm{~m} / \mathrm{s})$ (Figura $3 \mathrm{~b}$ ) e um aumento no fluxo de calor total que nessa fase tem valor de $100 \mathrm{~W} \mathrm{~m}^{-2}$ (Figura 2b), isso é uma característica importante, uma vez que a energia para o sistema é proveniente do oceano. As imagens de satélite (Figura 8b) mostram grandes áreas com nebulosidade próximo à região de ciclogênese indicando a manutenção da atividade convectiva.

Os fluxos de calor latente armazenam energia, que posteriormente liberam na atmosfera no processo de condensação. A condensação é máxima em níveis médios da atmosfera (Peixoto e Oort, 1992); por esse motivo, que o centro dos ciclones tropicais é bem mais quente do que seus arredores (Figura 7). Já os ventos mais intensos ocorrem próximo à superfície (Chavas et al., 2017) e tendem a decrescer com a altitude (Franklin, 2003; Giammanco et al., 2012). 

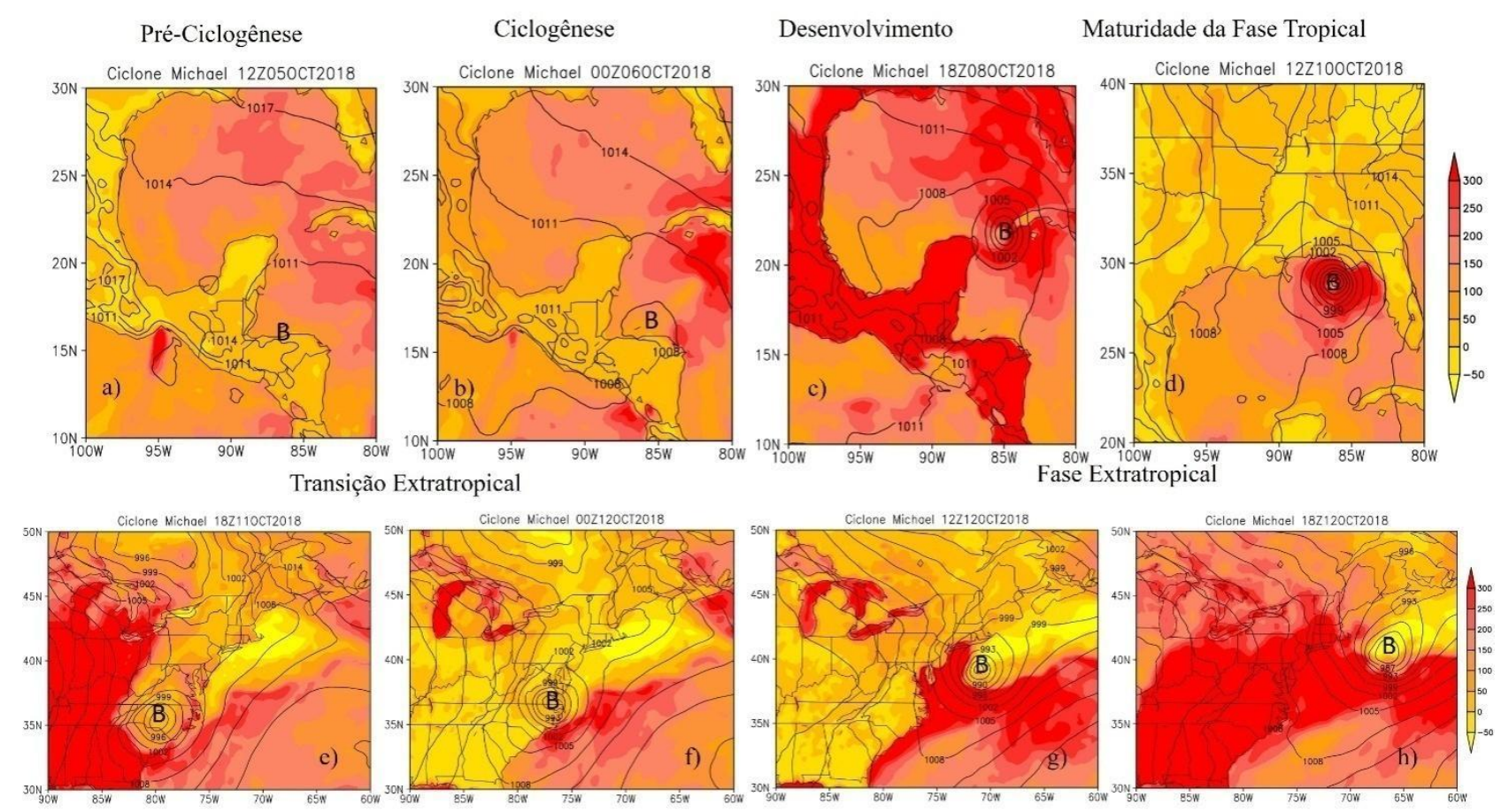

Figura 2. Fluxos de calor total (soma dos fluxos de calor latente e sensível; $\mathrm{W} / \mathrm{m}^{2}$ ) em colorido e pressão ao nível médio do mar (hPa, linhas pretas).a) pré-ciclogênese, b) ciclogênese, c) desenvolvimento, d) maturidade, (e-f) transição extratropical e (g-h) fase extratropical. Nas figuras, a letra B indica a localização do centro de baixa pressão.
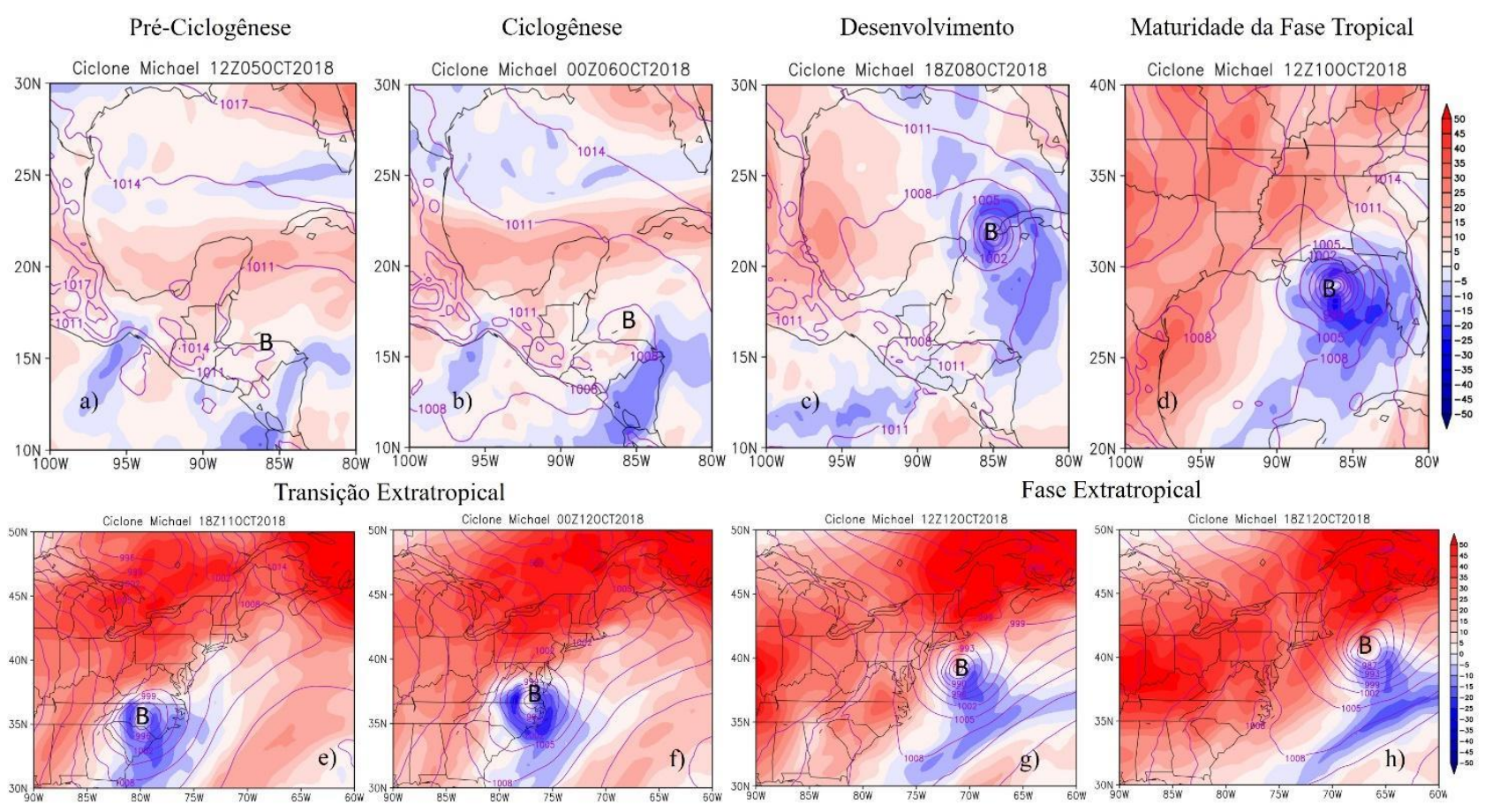

Figura 3. Cisalhamento vertical do vento entre 200 e $850 \mathrm{hpa}\left(\mathrm{m} \mathrm{s}^{-1}\right.$, preenchido) e pressão ao nível médio do mar (hpa, linhas roxas). a) pré-ciclogênese, b) ciclogênese, c) desenvolvimento, d) maturidade, (e-f) transição extratropical e (g-h) fase extratropical. Nas figuras, a letra B indica a localização do centro de baixa pressão. 
Após a gênese, o sistema torna-se autosuficiente: a baixa pressão em superfície (Figura 9c) induz a convergência dos ventos, favorecendo a transferência de fluxos de calor total do oceano para a atmosfera. Segundo Emanuel (1986), isso é um mecanismo de feedback positivo, pois à medida que a atmosfera recebe mais energia através dos fluxos de calor total, há mais energia para a atmosfera quando ocorre a condensação, assim o ar ascende ainda mais, tornando a pressão na superfície mais baixa, o que por sua vez contribui para a intensificação dos ventos em baixos níveis e esses para a transferência de energia mar-ar, logo o ciclone se torna mais intenso.
Do estágio de pré-ciclogênese para o atual, o tubo de vorticidade relativa torna-se mais definidoverticalmente (Figura 4c) e a nebulosidade mais simétrica (Figura 8c). A figura 7 mostra o desvio zonal da temperatura do ar e da altura geopotencial, nesta fase, o núcleo quente está mais definido e intenso a cerca de $250 \mathrm{hPa}$ (Figura 7c). $\mathrm{O}$ cisalhamento vertical do vento mostra valores negativos de $\sim-20 \mathrm{~m} / \mathrm{s}$ (Figura 3c), indicando que a intensidade dos ventos em altos níveis é menor do que a em baixos níveis, o que também foi observado em outros sistemas tropicais (Frank, 1977; Gray 1979; Franklin, 2003; Hart, 2003; He et al., 2016; Shu et al., 2017).
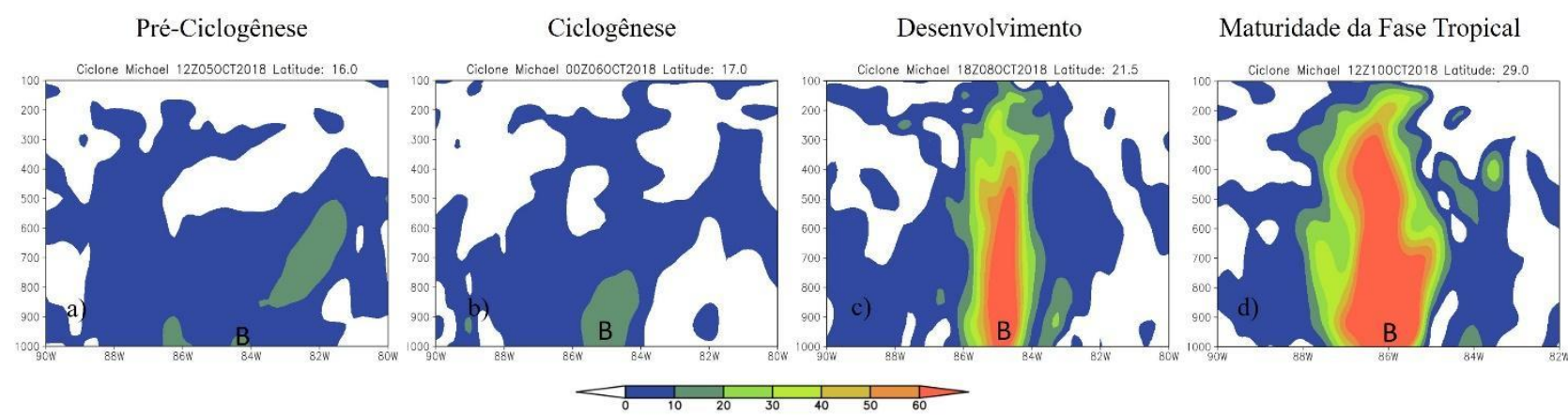

Transição Extratropical
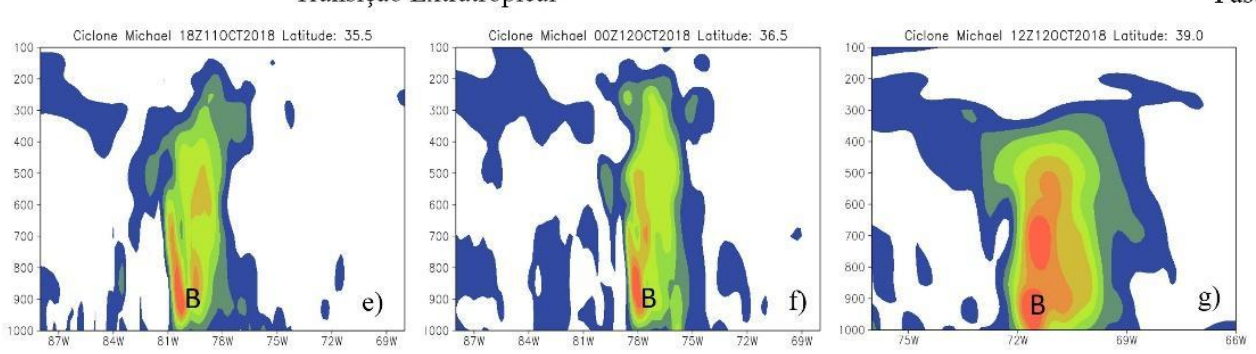

Fase Extratropical

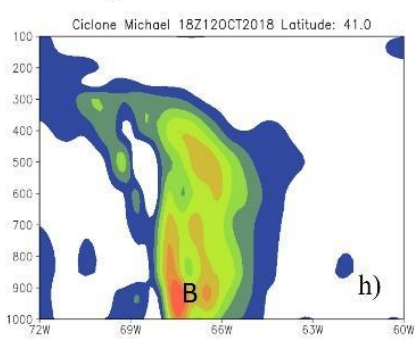

Figura 4. Perfil vertical da vorticidade relativa $\left(\mathrm{s}^{-1}\right)$. a) pré-ciclogênese, b) ciclogênese, c) desenvolvimento, d) maturidade, (e-f) transição extratropical e (g-h) fase extratropical. Nas figuras, a letra B indica a localização do centro de baixa pressão. 
Pré-Ciclogênese

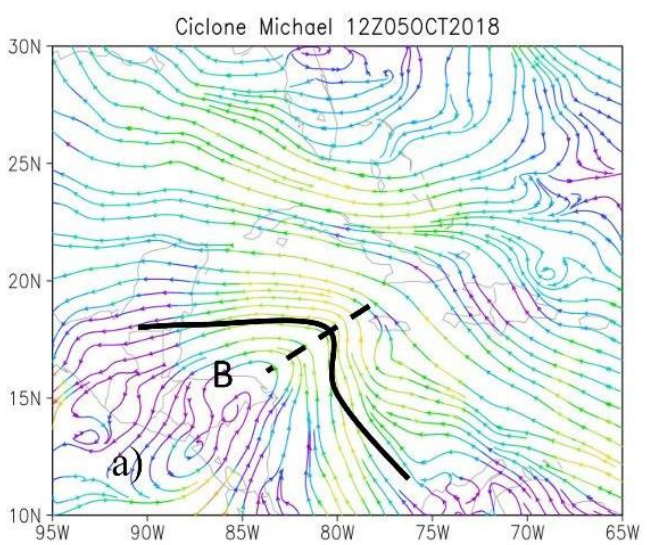

Ciclogênese

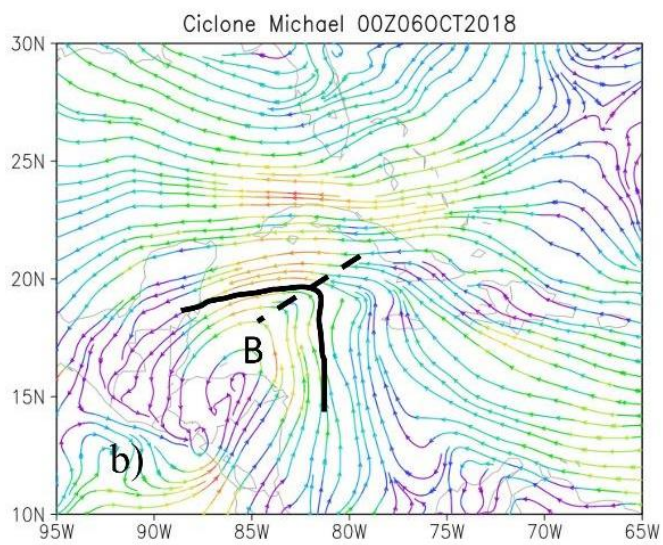

Figura 5. Linhas de corrente $\left(\mathrm{ms}^{-1}\right)$ em $850 \mathrm{hPa}$ no período de a) pré-ciclogênese e b) ciclogênese. Nas figuras, a letra B indica a localização do centro de baixa pressão.
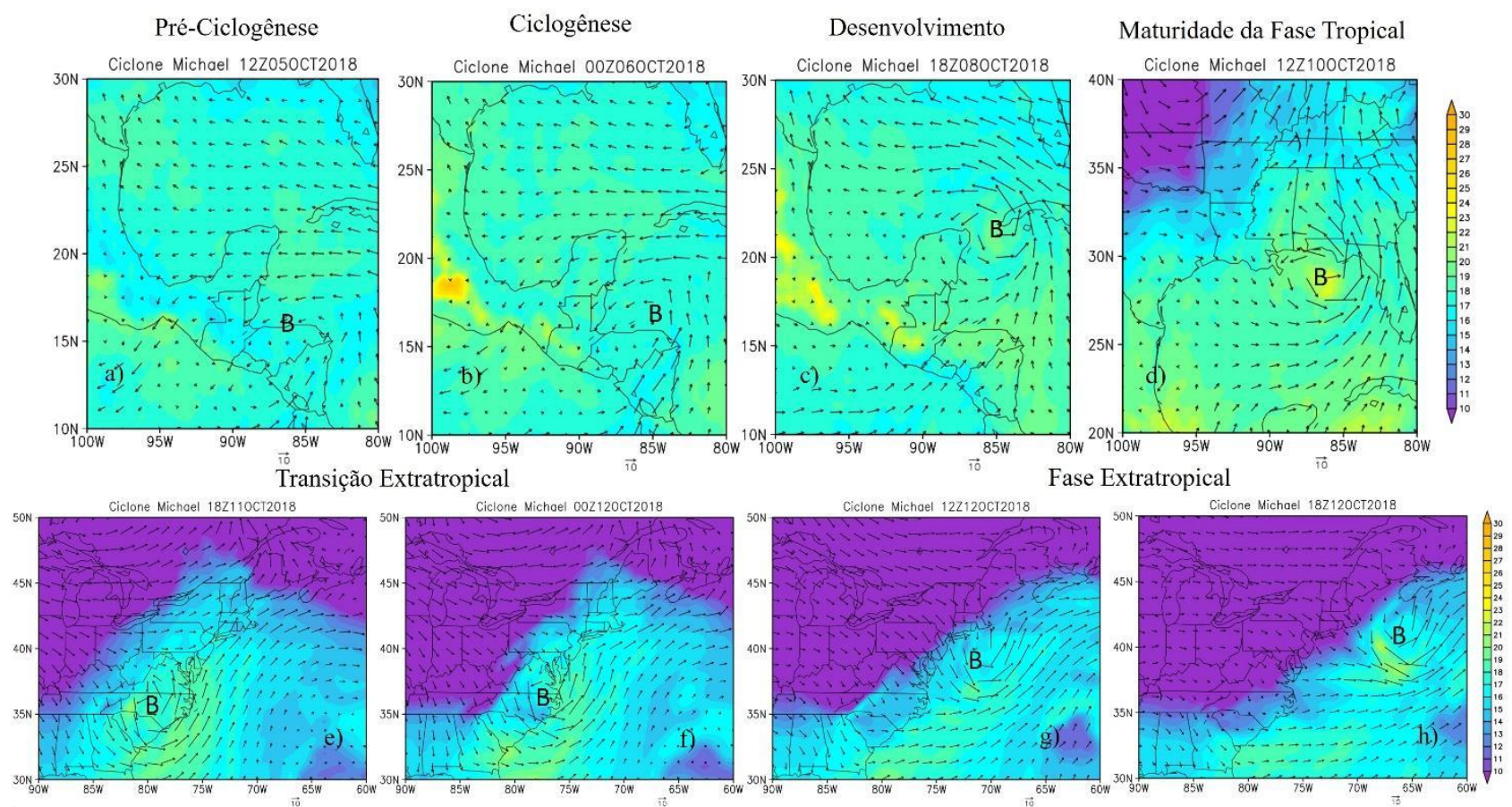

Figura 6. Temperatura do ar $\left({ }^{\circ} \mathrm{C}\right.$, preenchido) em $850 \mathrm{hPa}$ e vetores do vento $\left(\mathrm{m} \mathrm{s}^{-1}\right)$ em $850 \mathrm{hPa}$. a) préciclogênese, b) ciclogênese, c) desenvolvimento, d) maturidade, (e-f) transição extratropical e (g-h) fase extratropical. Nas figuras, a letra B indica a localização do centro de baixa pressão.

Pereira, R. G., Araujo, A. A., Reboita, M. S. 
Pré-Ciclogênese
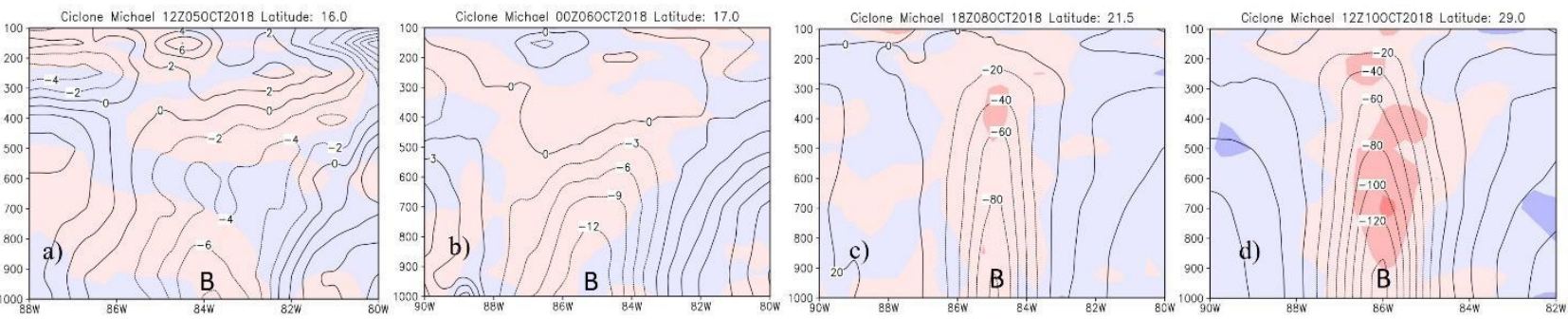

Transição Extratropical
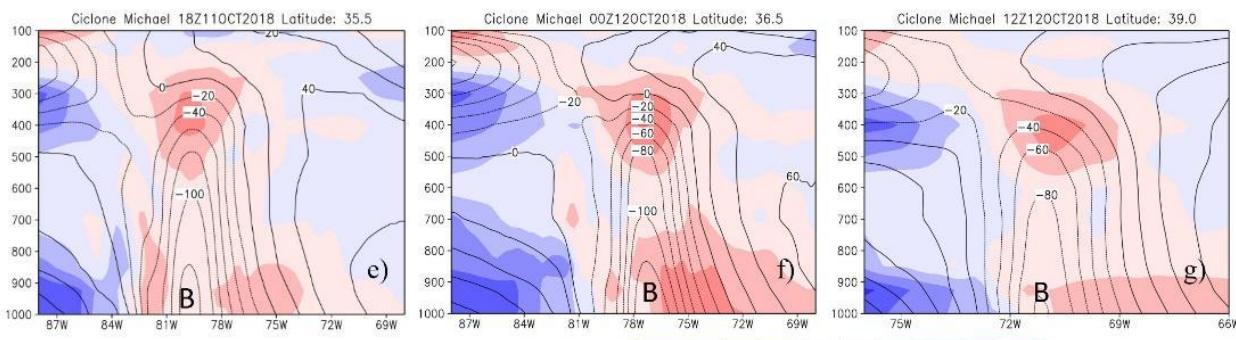

Fase Extratropical

Figura 7. Perfil vertical do desvio zonal da temperatura do $\operatorname{ar}\left({ }^{\circ} \mathrm{C}\right.$, preenchido) e da altura geopotencial (metros, linhas pretas). a) pré-ciclogênese, b) ciclogênese, c) desenvolvimento, d) maturid ade, (e-f) transição extratropical e (g-h) fase extratropical. Nas figuras, a letra B indica a localização do centro de baixa pressão.
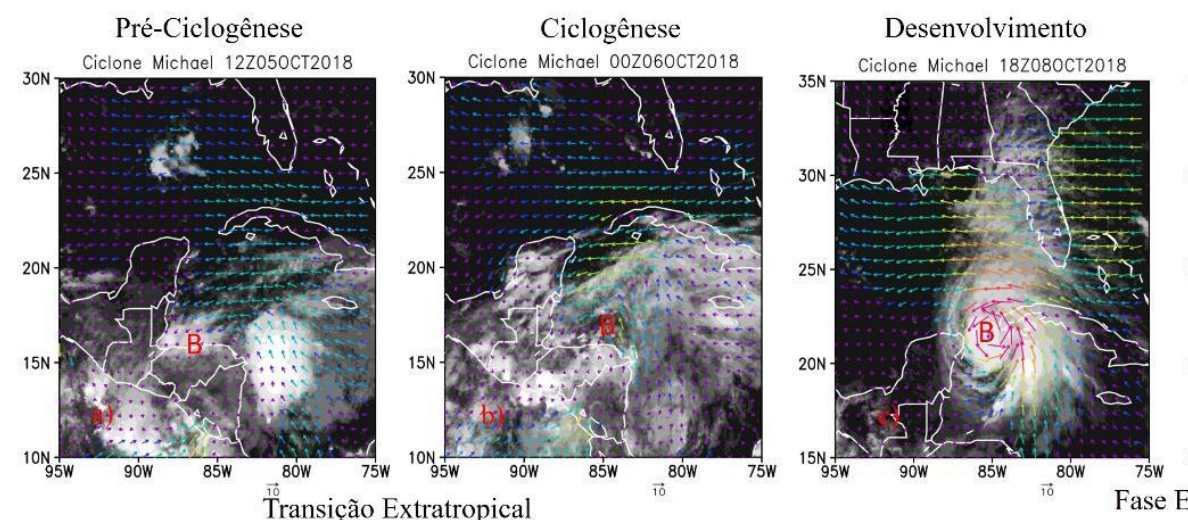

Maturidade da Fase Tropical

Transição Extratropical
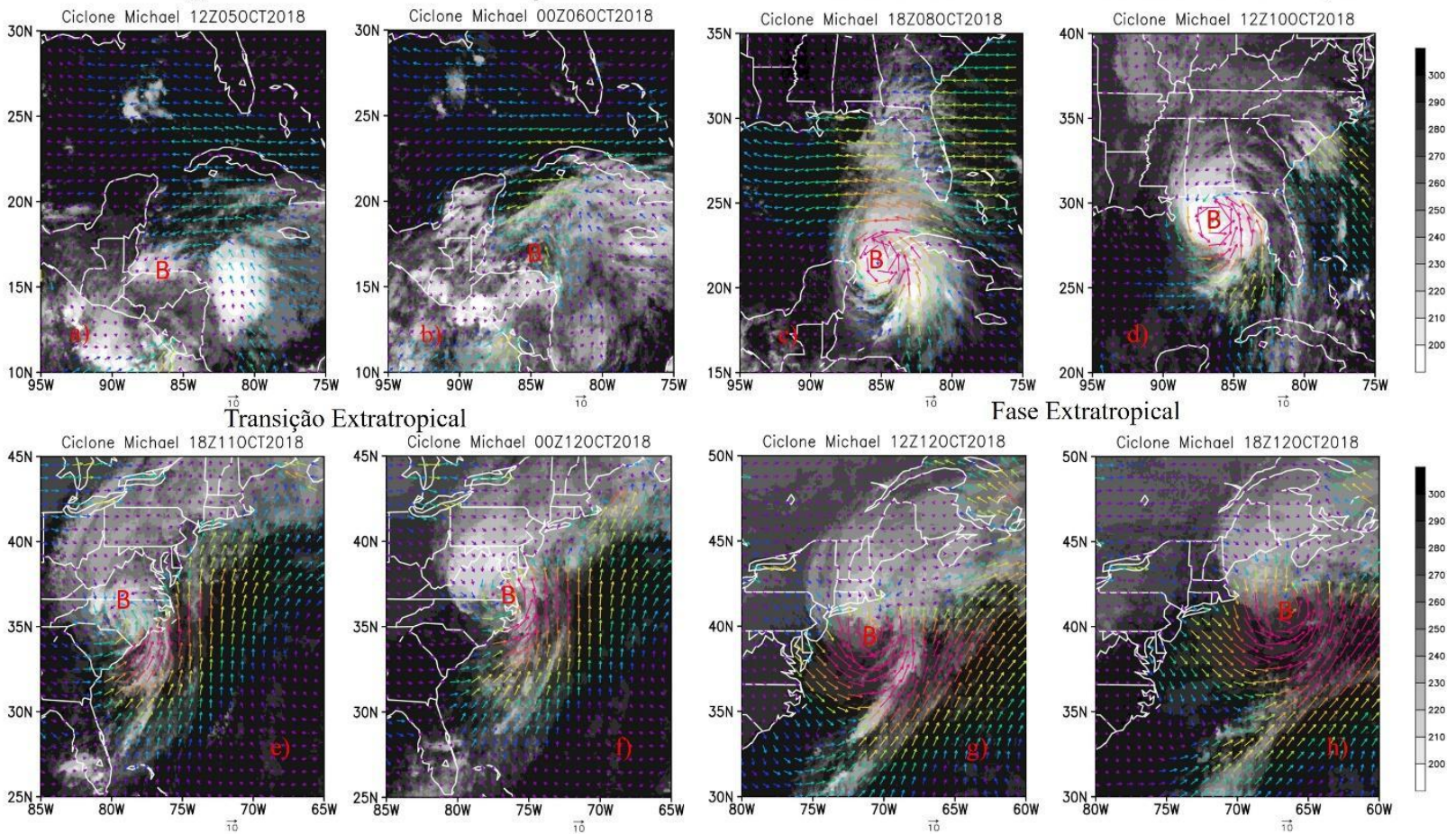

Figura 8. Temperatura de brilho $(\mathrm{K})$, vetores do vento a 10 metros $\left(\mathrm{m} \mathrm{s}^{-1}\right)$. a) pré-ciclogênese, b) ciclogênese, c) desenvolvimento, d) maturidade, (e-f) transição extratropical e (g-h) fase extratropical. Nas figuras, a letra $\mathrm{B}$ indica a localização do centro de baixa pressão. 

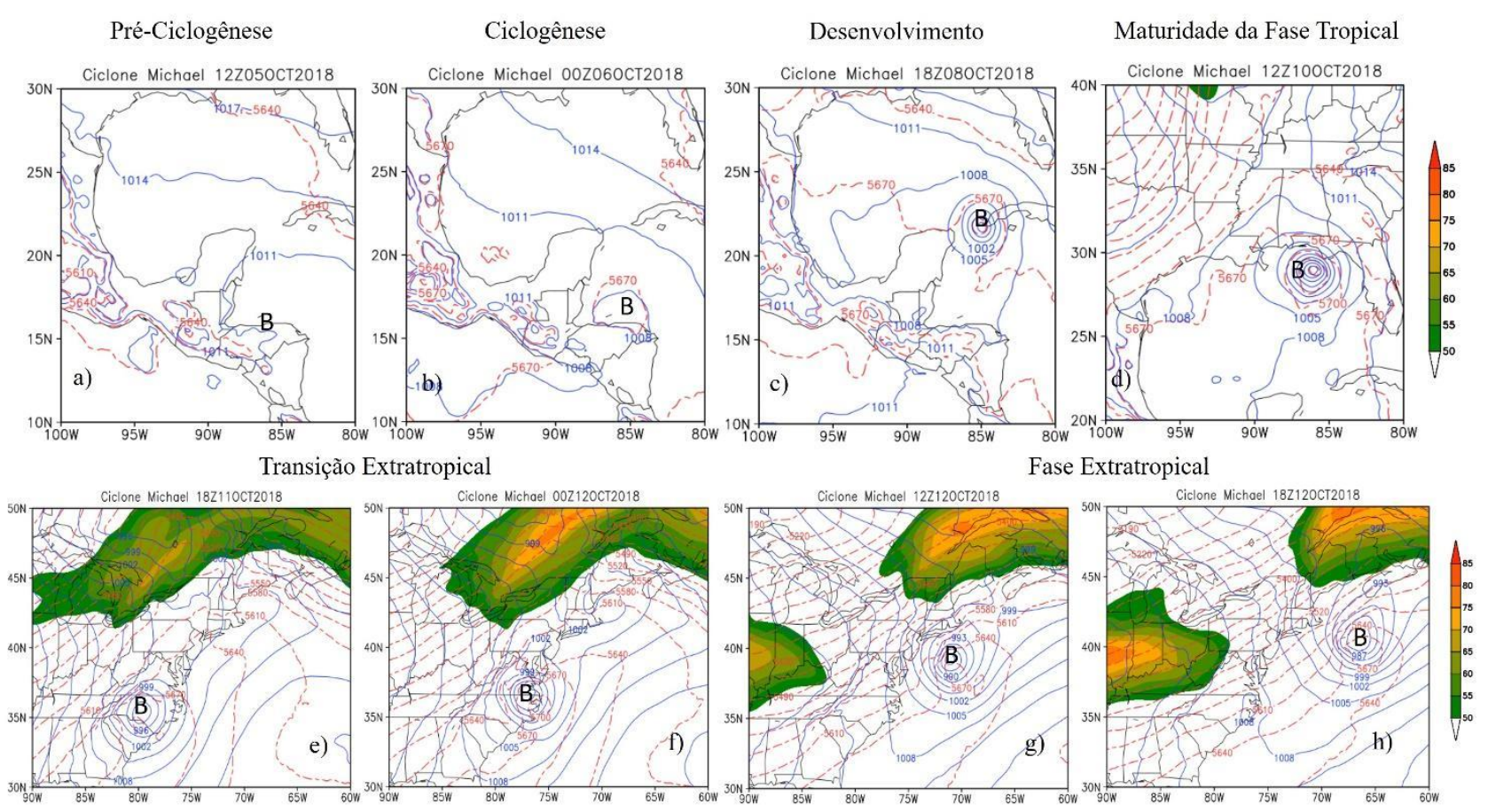

Figura 9. Pressão ao nível médio do mar (hPa, linhas azuis), espessura da camada 500/1000 hPa (metros, linhas vermelhas tracejadas) e intensidade do vento $\left(\mathrm{m} \mathrm{s}^{-1}\right)$ em $250 \mathrm{hPa}$ (preenchido). a) pré-ciclogênese, b) ciclogênese, c) desenvolvimento, d) maturidade, (e-f) transição extratropical e (g-h) fase extratropical. Nas figuras, a letra B indica a localização do centro de baixa pressão.

\section{Maturidade da fase tropical}

O dia 10 de outubro às 1200 UTC foi escolhido como estágio de maturidade, pois foi quando a vorticidade relativa ciclônica apresentou maior intensidade (Figura 4d).Ciclones tropicais apresentam, em geral, um olho em seu centro (Figura 10). Ao redor do olho, o círculo concêntrico de nuvens corresponde à parede do olho, podendo ter máxima intensidade convectiva (Emanuel, 2003).

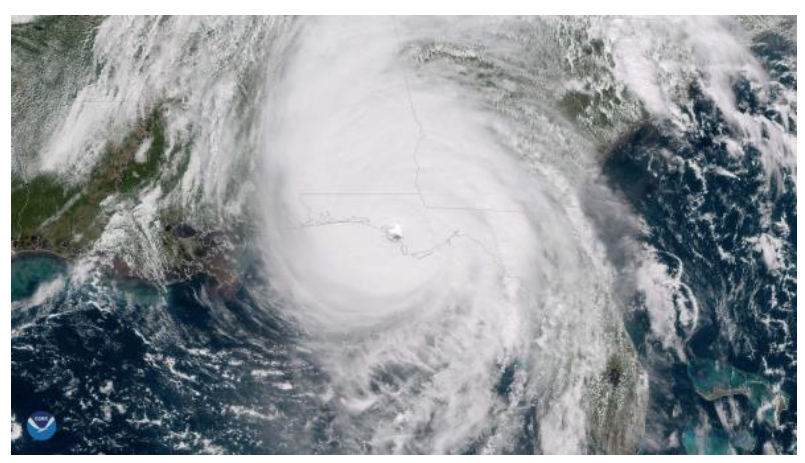

Figura 10. Imagem do satélite geoestacionário GOES-16 às 1730 UTC do dia 10 de outubro de 2018. Fonte: NOAA/NESDIS.

Com relação ao desvio zonal da temperatura do ar, o núcleo quente se expande ao longo das primeiras fases do ciclone, chegando a atingir níveis superiores a $200 \mathrm{hPa}$ na fase de maturação (Figura 7d). Nessa mesma fase, a vorticidade relativa atinge valores máximos de 60 s-1 (Figura 4d). Ao contrário do desvio zonal de temperatura, o desvio zonal de altura geopotencial mostra valores negativos (Figura 7d), pois sendo o ciclone um centro de baixa pressão, essa decresce mais rápido com a altura (Wallace e Hobbs, 2006).

\section{Transição Extratropical}

A transição extratropical é o processo associado à transformação de um ciclone tropical em um ciclone extratropical uma vez que o sistema tropical encontraum ambiente baroclínico e com redução da TSM (Evans et al., 2017). No dia 12 de outubro às 0000 UTC o valor da TSM na região do ciclone era de $26^{\circ} \mathrm{C}$ (Figura não mostrada). Esse valor embora seja propício para sistemas tropicais, não pode manter o sistema com tais características em virtude dos gradientes horizontais de temperatura do ar mostrados pelas linhas de espessura (Figuras 9e e 9f) e pelo campo de temperatura em $850 \mathrm{hPa}$ (Figuras 6e e 6f). A transição ex tratropical do ciclone Michael iniciou quando ele se moveu para a Carolina do Norte, com a pressão caindoe os ventos se intensificando a oeste e noroeste do sistema. No estágio de 
transição há um enfraquecimento da vorticidade relativa ciclônica (Figuras 4e e 4f), do desvio zonal negativo de altura geopotencial e do núcleo quente do desvio zonal da temperatura (Figuras $7 \mathrm{e}$ e 7f) quando comparados ao estágio anterior. Num ambiente baroclínico, o cisalhamento vertical do vento é mais intenso. Isso pode ser observado ao se comparar a fase de transição com as anteriores. $\mathrm{Na}$ transição, o cisalhamento continua negativo apenas no centro do ciclone (Figuras $3 \mathrm{e}$ e $3 \mathrm{f}$ ). Os valores de calor total diminuem em relação a fase anterior (Figuras 2e e 2f).

Muitos ciclones tropicais que se movem para latitudes médias e se transformam em ciclones extratropicais, geralmente, causam chuvas intensas e ventos fortes, o que ameaça as atividades terrestres e marítimas (Jones et al., 2003). Sendo um ciclone de categoria 5, no caso do Michael ocorreram ventos fortes, principalmente sobre a baía inferior de Chesapeake e nas áreas adjacentes. Ventos intensos também atingiram a região da costa leste dos EUA até o norte do sul de Nova Jersey, à medida que Michael migrava para o mar, o que também está descrito em Beven II et al. (2019).

\section{Fase Extratropical}

Finalizada a transição, às 1200 UTC do dia 12 de outubro se inicia a fase extratropical do ciclone Michael. Os valores de cisalhamento (Figuras $3 \mathrm{~g} \mathrm{e} 3 \mathrm{~h}$ ) e fluxos de calor total (Figuras $2 \mathrm{~g}$ e $2 \mathrm{~h}$ ) nessa fase aumentaram em relação ao estágio anterior. A nebulosidade (que no estágio tropical era simétrica) se desconfigura ainda mais e há nuvens menos espessas (Figuras 8g e 8h). Após esse horário o sistema se mantém até o dia 15 quando se desconfigura.

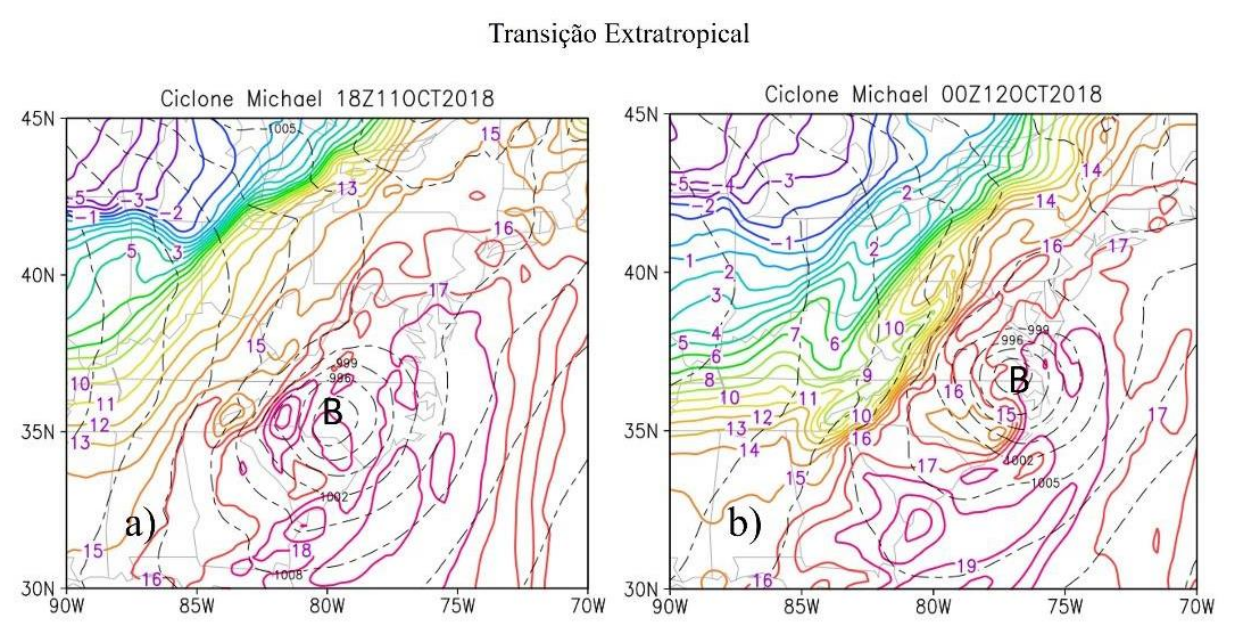

Figura 11. Isotermas em $850 \mathrm{hPa}\left({ }^{\circ} \mathrm{C}\right.$, linhas coloridas) e a pressão ao nível médio do mar (hPa, linhas tracejadas) no período de transição extratropical. Nas figuras, a letra B indica a localização do centro de baixa pressão.

\section{Conclusão}

Michael foi um ciclone de categoria 5, que produziu ventos devastadores e chuvas fortes o que causou 16 mortes e cerca de US \$ 25 bilhões em danos nos Estados Unidos (Beven II et al., 2019).

Este trabalho teve como objetivo descrever o ciclo de vida do Michael, com destaque na fase de gênese e transição extratropical. O ambienteem que ocorreu a gênese foi caracterizado por temperatura da superfície do mar (TSM) de $29^{\circ} \mathrm{C}$, por fluxos de calor total (sensívele latente) da superfície oceânica para a atmosfera variando entre $\sim 50 \mathrm{~W} \mathrm{~m}^{-2}$, cisalhamento vertical do vento de $\sim 0 \mathrm{~m} / \mathrm{se}$ fraca vorticidade relativa ciclônica $\left(10 \mathrm{~s}^{-1}\right)$ em baixos níveis da atmosfera (nível de $980 \mathrm{hPa}$ ) na região em que o ciclone se forma. Essas características seguem as definidas por Gray (1968).

O dia 6 de outubro às 0000 UTC foi definido como ciclogênese, pois foi quando as isóbaras apareceram fechadas pela primeira vez, com núcleo possuindo $1008 \mathrm{hPa}$ e vorticidade relativa ciclônica mostrando assinatura no perfil vertical.

O dia 10 de outubro às 1200 UTC indica o estágio de maturidade, poisfoi quando a vorticidade relativa ciclônica apresentou maior intensidade. Os perfis verticais mostramum alinhamento vertical do tubo de vorticidade relativa ciclônica, do desvio zonal negativo de altura geopotencial e positivo de temperatura do ar. 
Quando o ciclone Michael se deslocou para latitudes médias, se transformou em ciclone extratropical. No estágio de transição há um enfraquecimento da vorticidade relativa ciclônica, do desvio zonal negativo de altura geopotencial e do núcleo quente do desvio zonal da temperatura quando comparados ao estágio anterior, e a nebulosidade adquiriu um padrão menos simétrico. Quando transcionou provocou ventos fortes e chuvas intensas, ameaçando as atividades e vidas da população, reforçando desta maneira a importância de estudar tais sistemas.

\section{Agradecimentos}

Aos centros de meteorologia que disponibilizaram os dados para o estudo e ao CNPq.

\section{Referências}

Anthes, R.A., 1982. Tropical Cyclones. Their Evolution, Structure and Effects, 1 ed. Amer Meteorological Society.

Beven II, J.L., Berg, R., Hagen, A., 2019. Hurricane Michael. National Hurricane Center. Disponível:

https://www.nhc.noaa.gov/data/tcr/AL142018_ Michael.pdf .Acesso: 10 abr. 2020.

Bister, M., Emanuel, K.A., 1998. Dissipative heating and hurricane intensity. Meteorology and Atmospheric Physics 65, 233-240. https://doi.org/10.1007/BF01030791.

Bjerknes, J., Solberg, H., 1922. Life cycle of cyclones and the polar front theory of atmospheric circulation. Geophysisks Publikationer 3, 3-18. https://doi.org/10.1002/qj.49704920609

Charney, G. J., Ellassen, A., 1964. On the South of the Hurricane Depression. Journal of the atmospheric sciences 21, 68-75.

Chavas, D.R., Reed, K.A., Knaff, J.A., 2017. Physical understanding of the tropical cyclone wind-pressure relationship. Nature communications $\quad 8, \quad 1360$. https://doi.org/10.1038/s41467-017-01546-9

C3S. Copernicus Climate Change Service. ERA5: Fifth generation of ECMWF atmospheric reanalyses of the global climate, Copernicus Climate Change Service Climate Data Store (CDS),

2020. Disponível:https://cds.climate.copernicus.eu/. Acesso: 01 mai. 2020.

Emanuel, K.A., 1986. An air-sea interaction theory for tropical cyclones. Part I: Steady state maintenance. Journal of Atmospheric Sciences
43 , $585-604$. https://doi.org/10.1175/15200469(1986)043<0 585:AASITF $>2.0 . \mathrm{CO} ; 2$.

Emanuel, K.A., 2003. Tropical Cyclones. Annual Review of Earth and Planetary Sciences 31, 75 104.https://doi.org/10.1146/annurev .earth.31.10 0901.141259

Evans, C., Wood, K. M., Aberson, S. D., Archambault, H. M., Milrad, S. M., Bosart, L. F., Corbosiero, K. L., Davis, C. A., J. R.D., Pinto,Doyle, J., Fogarty, C., Galarneau, T. J.,Grams, C. M., Griffin, K. S., Gyakum, J., Hart, R. E., Kitabatake, N., Lentink, H. S., McTaggart-Cowan, R., Perrie, W., Quinting, J. F., Reynolds, C. A., Riemer, M., Ritchie, E. A., Sun, Y. Zhang, F., 2017. The extratropical transition of tropical cyclones. Part I: Cyclone evolution and direct impacts. Monthly Weather Review 145, 4317-4344. https://doi.org/10.1175/MWR-D-17-0027.1.

Frank, W.M., 1977. The structure and energetics of the tropical cyclone I. Storm structure. Monthly Weather Review 105, 1136-1150. https://doi.org/10.1175/15200493(1977)105<11 36:TSAEOT $>2.0 . \mathrm{CO} ; 2$

Franklin, J.L., Black, M.L., Valde, K., 2003. GPS dropwind- sonde wind profiles in hurricanes and their operational implications. Weather and Forecasting $18, \quad 32-44$. https://doi.org/10.1175/15200434(2003)018<0 032:GDWPIH > 2.0.CO;2

Giammanco, I.M., Schroeder, J.L., Powell, M.D., 2012. Observed characteristics of tropical cyclone vertical wind profiles. Wind and Structures $\quad 15, \quad 65-86$. https://doi.org/10.12989/was.2012.15.1.065

Gray, W.M., 1967. Global view of the origin of tropical disturbances and storms. Colorado State University, Department of Atmospheric Science.

Gray, W.M., 1968. Global view of the origin of tropical disturbances and storms. Monthly Weather Review 96, 669-700. https://doi.org/10.1175/15200493(1968)096<0 669:GVOTOO $>2.0 . \mathrm{CO} ; 2$

Hart, R.E., 2003. A cyclone phase space derived from thermal wind and thermal asymmetry. Monthly Weather Review 131, 585-616. https://doi.org/10.1175/15200493(2003)131<0 585:ACPSDF> 2.0.CO;2

Hart, R. E., Evans, J. L., Evans, C., 2006. Synoptic composites of the extratropical transition life cycle of North Atlantic tropical cyclones: Factors determining posttransition evolution. Monthly Weather Review 134, 553-578. https://doi.org/10.1175/MWR3082.1 
He, Y.C., Chan, P.W., Li, Q.S., 2016. Observations of vertical wind profiles of tropical cyclones at coastal areas. Journal of Wind Engineering and Industrial Aerodynamics 152, 1-14. https://doi.org/10.1016/j.jweia.2016.01.009

Jones, S.C., Haar, P.A., Abraham, J., Bosart, L.F. et al., 2003. The Extratropical Transition of Tropical Cyclones: Forecast Challenges, Current Understanding, and Future Directions. Weather and Forecasting 18, 1052-1092. https://doi.org/10.1175/15200434(2003)018<10 52:TETOTC $>2.0 . \mathrm{CO} ; 2$

Knapp, K. R., Ansari, S., Bain, C. L., Bourassa, M. A., Dickinson, M. J., Funk, C., Helms, C. N., Hennon, C. H., Holmes, C. D., Huffman, G. J., Kossin, J. P., Lee, H.T., Loew, A., Magnusdottir, G., 2011. Globally gridded satellite (GridSat) observations for climate studies. Bulletin of the American Meteorological Society 92, 893-907.

Lim, Y.K., Schubert, S.D., Reale, O., Lee, M.I., Molod, A.M., Suarez, M.J., 2015. Sensitivity of tropical cyclones to parameterized convection in the NASA GEOS-5 model. Journal of Climate 28, 551-573.

NHC. National Hurricane Center, 2020. Disponível: https://www.nhc.noaa.gov. Acesso em: 20 abr. 2020.

Peixoto, P.J., Oort, A.H., 1992. Physics of climate, 1 ed. American Institute of Physics, New York.

Reboita, M.S., Gan, M.A., Rocha, R.P., Custodio, I.S., 2017a. Ciclones em Superfície nas Latitudes Austrais: Parte I. Revisão Bibliográfica. Revista Brasileira de Meteorologia 32, 171-186. https://doi.org/10.1590/0102-77863220010.
Reboita, M.S., Gan, M.A., Rocha, R.P., Custodio, I.S., 2017b. Ciclones em Superfície nas Latitudes Austrais: Parte II. Estudo de Casos. Revista Brasileira de Meteorologia 32,509-542. https://doi.org/10.1590/0102-7786324002.

Ritchie, E.A., Esberry, R.L., 2006. Simulations of the Extratropical Transition of Tropical Cyclones: Phasing between the Upper-Level Trough and Tropical Cyclones. Monthly weather review $135,862-876$. https://doi.org/10.1175/MWR3303.1

Shu, Z.R., Li, Q.S., He, Y.C., Chan, P.W., 2017. Vertical wind profiles for typhoon, monsoon and thunderstorm winds. Journal of Wind Engineering and Industrial Aerodynamics 168, 190-199. https://doi.org/10.1016/j.jweia.2017.06.004

Takamura, N., Wada, A., 2020. Unusual Characteristics of Extratropical Transition of 3 Typhoons in August 2016. Journal of the Meteorological Society of Japan [online] 98. Disponível:

https://www.jstage.jst.go.jp/article/jmsj/advpub /0/advpub_2020-035/_pdf. Acesso: 15 mai. 2020.

Wallace, J.M., Hobbs, P.V., 2006. Atmospheric science: an introductory survey. University of Washington.

Wang, Y., Wu, C.C., 2004. Current understanding of tropical cyclone structure and intensity changes - a review. Meteorology and Atmospheric Physics. 87, 257-278. https://doi.org/10.1007/s00703-003-0055-6. 